\title{
RELAÇÃO DOS SOLOS NA QUANTIDADE DE SÓLIDOS EM ÁGUAS SUPERFICIAIS - O CASO DA BACIA HIDROGRÁFICA DO RIO PIRAPÓ, PR
}

\author{
Cássia Maria Bonifácio
}

Célia Regina Granhen Tavares ${ }^{2}$

Diogo Yukio Uema ${ }^{3}$

\begin{abstract}
RESUMO
Desde 1999, com a Política Estadual de Recursos Hídricos, o Estado do Paraná incentiva o desenvolvimento de pesquisas voltadas à recuperação de mananciais de bacias hidrográficas. Algumas bacias hidrográficas já possuem seus estudos concluídos, dentre as quais é possível citar a bacia do Alto do Iguaçu e rio Ribeira, do Tibagi e do rio Jordão. Os resultados apresentados pelas pesquisas corroboraram com a elaboração dos respectivos Planos de Bacia. Todavia é importante ressaltar que a bacia hidrográfica do rio Pirapó ainda caminha em relação à elaboração deste Plano, o que torna necessário o monitoramento desta bacia, para a compreensão de seu funcionamento estrutural. Tendo em vista que os dados resultantes sirvam de auxílio na tomada de decisões.O trabalho teve como objetivo principal monitorar o parâmetro de sólidos dissolvidos e suspensos, presentes nas águas superficiais de determinados pontos, na bacia do rio Pirapó - PR, levando em consideração a relação da geologia e tipo de solo. O método utilizado foi o gravimétrico, e a unidade de medida é expressa em $\mathrm{mg} \mathrm{L}^{-1}$. Os resultados demonstraram que, para os parâmetros de sólidos dissolvidos e suspensos, os menores valores foram encontrados nos pontos 1 e 2, alocados sobre Basalto, com Latossolo Vermelho e Nitossolo, textura argilosa, e, os resultados mais elevados nos pontos 3 e 4, sobre os Depósitos Sedimentares, ou seja, estão associados à solos Latossolo Vermelho e Argissolo Vermelho, ambos de textura média; e em relação ao ponto 5, este apresenta resultados mais elevados que os dos pontos 1 e 2, mesmo ambos estando sobre o Basalto, todavia isso se justifica por sua localização, a jusante da bacia.
\end{abstract}

PALAVRAS-CHAVE: Qualidade da água, rio Pirapó, Solos.

\footnotetext{
${ }^{1}$ Mestre em Geografia, UEM.

${ }^{2}$ Prof. Dra. do Departamento de Engenharia Química, UEM.

${ }^{3}$ Graduando em Geografia, UEM
} 


\title{
SOILS OF RELATIONSHIP IN THE AMOUNT OF SOLID SURFACE WATER - The RIVER BASIN CASE OF RIO Pirapó, $P R$
}

\begin{abstract}
Since 1999, with the State Water Resources Policy, the State of Paraná encourages the development of research aimed at recovery watershed sources. Some watersheds already have their completed studies, among which we can mention the High Basin of Iguaçu River and Ribeira, the Tibagi and the Jordan River. The results presented by the research corroborated with the preparation of their Basin Plans. However it is worth noting that the catchment area of the river Pirapó still walks in relation to the preparation of this Plan, which calls for the monitoring of the basin, to understand its structural function. Considering that the resulting data will serve as aid in making decisões. $O$ work aimed to monitor the dissolved solids and suspended parameter, present in surface waters of certain points in the basin of river Pirapó - PR, taking into account the relationship of geology and soil type. The method used was the gravimetric, and the unit of measure is expressed in $m g$ L-1. The results showed that, for the parameters of dissolved and suspended solids, the lowest values were found in sections 1 and 2, allocated on Basalt, with Rhodic and Nitosol, clayey, and the highest results in paragraphs 3 and 4, on Sedimentary deposits, ie are associated with soil Oxisol and Ultisol, both of medium texture; and in relation to paragraph 5, this shows higher results than the points 1 and 2, even both standing over Basalt, however it is justified by its location downstream of the basin.
\end{abstract}

KEYWORDS: Water quality, river Pirapó, Soils.

\section{RELACIÓN DE SUELOS EN LA CANTIDADE DE SÓLIDOS EN AGUAS SUPERFICIALES - EL CASO DE LA CUENCA DEL DO RIO PIRAPÓ, PR}

\begin{abstract}
RESUMEN
Desde 1999, con la Política de Estado sobre Recursos Hídricos, el Estado de Paraná fomenta el desarrollo de la investigación dirigida a la recuperación de fuentes de las cuencas hidrográficas. Algunas cuencas ya tienen estúdios completados, entre las cuales es posible mencionar la cuenca del Alto do Iguaçú y el río Ribeira, río Tibagi y el río Jordão. Los resultados presentados por la investigación corroboran con la preparación de sus planes de cuenca.

Sin embargo, es importante destacar que la cuenca del río Pirapó todavía camina en relación con la preparación de este Plan, que requiere el monitoramiento de la cuenca, para la comprensión de su función estructural. Dado que los datos resultantes sirven para ayudar en la toma de decisiones.

El trabajo tuvo como objetivo monitorear el parámetro de sólidos suspendidos y disueltos, presentes en las aguas superficiales de algunos puntos de la cuenca del río Pirapó - relaciones públicas, teniendo en cuenta la relación de la geología y tipo de suelo.

Se utilizó el método gravimétrico, y la unidad de medida se expresa en $\mathrm{mg} \mathrm{L}^{-1}$. Los resultados mostraron que, para los parámetros de sólidos disueltos y suspendidos, se encontraron los valores más bajos en 1 y 2, ubicados en Basalto con Latossolo Vermelho e Nitossolo, arcillosos, y los más altos resultados en las secciones 3 y 4, em los Depósitos Sedimentarios, o sea, están asociados con los suelos Latossolo Vermelho y Argissolo Vermelho, de textura media; y en relación con la sección 5, esto demuestra resultado mayor que de los puntos 1 y 2 , mismo estando en el basalto, sin embargo, esto se justifica por su ubicación aguas abajo de la cuenca .
\end{abstract}

PALABRAS-CLAVE: Calidade el agua, río Pirapó, Suelos. 


\section{INTRODUÇÃO}

As mudanças ambientais, ocasionadas pela ação antrópica, como a erosão e a poluição têm se intensificado a nível mundial (FREIRE, 2010).

A erosão do solo pode ser entendida como sendo o transporte de partículas de solo, que são carreadas para os corpos d'água, em eventos de precipitação. E está relacionada ao tipo de solo, quantidade de água precipitada, declividade do terreno e principalmente ao uso da terra (BONIFÁCIO, 2013).

O monitoramento da qualidade da água via parâmetros físicos, como presença de sólidos, turbidez e cor, num intervalo temporal, fornece dados a respeito das características das atividades predominantes em determinada bacia, o que contribui ao maior ou menor carreamento superficial de sedimentos até a calha do curso d'água principal (TARELHO, BONIFÁCIO, TAVARES, 2013).

Conforme os estudos de Bertoni e Neto (1999) a água superficial, que não pode ser infiltrada, é o maior agente transportador de partículas do solo. O que pode ocasionar também no transporte de poluentes, ligados a essas partículas. Ou seja, a poluição pode ser provocada por ação antrópica ou por ação natural (BRAGA, 2005).

Para Von Sperling (2007), a qualidade da água é resultante de fenômenos naturais e da ação antrópica.

Os agentes naturais são compostos inorgânicos, responsáveis pela coloração das águas, cujas propriedades podem provocar os efeitos de matéria em estado coloidal. Dentre estes, é possível citar os óxidos de ferro e manganês, que são abundantes em diversos tipos de solos, inclusive na área de estudo, a bacia hidrográfica do rio Pirapó - PR. Todavia, solos originados por rochas sedimentares, apesar de não apresentarem coloração evidente, pela falta de óxidos, estão presentes em maior quantidade, pela questão de sua textura, que é mais propícia à lavagem, promovida pela precipitação.

Em relação à área de estudo, a bacia tem sua nascente em área urbana, todavia, ao longo de seu trajeto, apresenta usos da terra misto, urbano e rural. Nas áreas urbanas é possível destacar o carreamento de partículas devido à intensa impermeabilização do solo, já em meio rural, destinado à produção agrícola intensiva, 
ocorre à degradação dos atributos físicos do solo, resultando também no aporte de materiais sólidos e outras substâncias para o curso d'água (SALA, 2005; SCHNEIDER, 2009; FREIRE, 2010).

Com esse pressuposto, foi dado enfoque à questão da interferência de fenômenos naturais, na qualidade da água, por meio dos sólidos dissolvidos e suspensos, levando em consideração a relação o tipo de solo, presentes ao longo da bacia hidrográfica, uma vez que os pontos selecionados pertencem ao mesmo tipo de uso da terra.

\section{DESENVOLVIMENTO}

Para representar satisfatoriamente a bacia hidrográfica do rio Pirapó (Figura 1), foram coletadas amostras de água em cinco pontos, já estipulados (Tabela 1), conforme a classificação em unidades de paisagem e Formação geológica, o que se justifica pela diferença entre os compartimentos locais e tipos de solo relacionados à geologia. 
Figura 1: Bacia hidrográfica do rio Pirapó - PR.

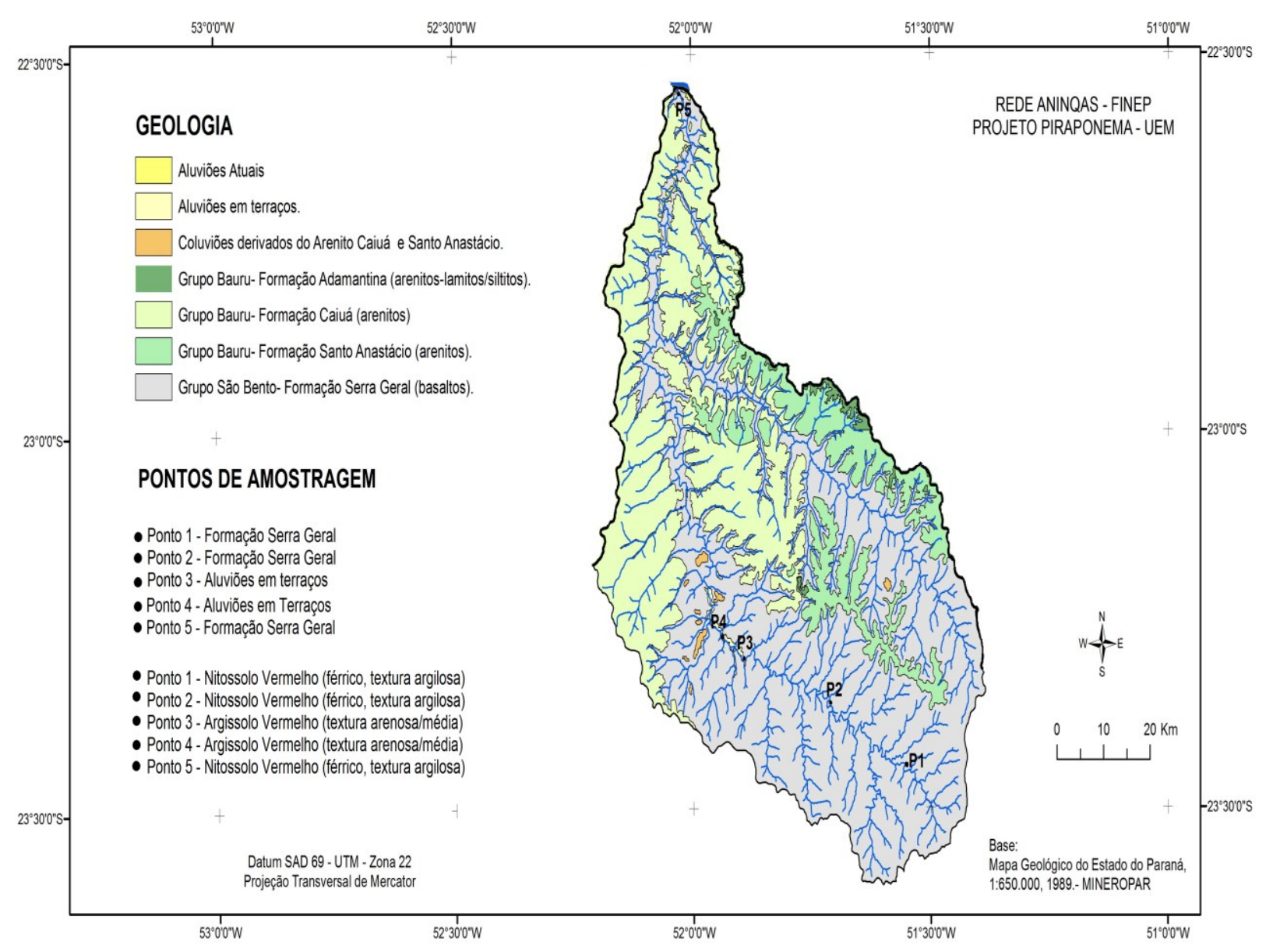

Fonte: Rede ANINQAS / Projeto Piraponema (2012). 
Tabela 1: Localização dos pontos de amostragem na bacia hidrográfica do rio Pirapó - PR.

\begin{tabular}{|c|c|c|c|c|c|}
\hline & Pontos & Local & Latitude & Longitude & Altitude (m) \\
\hline \multirow{4}{*}{ 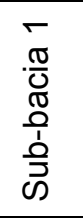 } & 1 & A jusante do córrego Água do Abutre & $23^{\circ} 26^{\prime} 59^{\prime \prime S}$ & $51^{\circ} 33^{\prime} 19^{\prime \prime} \mathrm{W}$ & 553 \\
\hline & 2 & A jusante do ribeirão Araçu & $23^{\circ} 21^{\prime} 96^{\prime \prime S}$ & $51^{\circ} 42^{\prime} 51^{\prime \prime} \mathrm{W}$ & 411 \\
\hline & 3 & A jusante do ribeirão Guarujá & $23^{\circ} 18^{\prime} 25^{\prime \prime S}$ & $51^{\circ} 53^{\prime} 59^{\prime \prime} \mathrm{W}$ & 393 \\
\hline & 4 & A jusante do ribeirão Maringá & $23^{\circ} 17^{\prime} 19^{\prime \prime S}$ & $51^{\circ} 55^{\prime} 42^{\prime \prime} \mathrm{W}$ & 394 \\
\hline 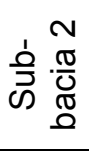 & 5 & A jusante do ribeirão Água do Pau d'Alho & $22^{\circ} 32^{\prime} 90 " \mathrm{~S}$ & $52^{\circ} 01^{\prime} 72^{\prime \prime} \mathrm{W}$ & 269 \\
\hline
\end{tabular}

As coletas seguiram uma frequência mensal ao longo do projeto, referente ao período de janeiro a agosto de 2014. As amostras foram coletadas de forma pontual, no ponto central da seção do curso d'água, e armazenadas em frascos de polietileno (2L) e mantidas em resfriamento. A sistemática de preservação das amostras seguiu a metodologia proposta no Standard Methods for Examination of Water and Wastewater (APHA, 1998).

As análises foram realizadas no Laboratório de Gestão, Preservação e Controle Ambiental (LGPCA) do Departamento de Engenharia Química da Universidade Estadual de Maringá (UEM). Dentre os parâmetros realizados estão: sólidos em suspensão e sólidos totais. Para garantir confiabilidade dos resultados obtidos, os testes foram executados em duplicata, possibilitando o cálculo do resultado médio.

A experimentação é parte do projeto de Monitoramento de Bacias Urbanas e Rurais, Análise Integrada da Qualidade da Água e Aspectos Sócio-Econômicos, e está financiado pelo CNPQ, por projeto sob a responsabilidade da Prof. Dra. Célia Regina Granhen Tavares.

O método utilizado foi o gravimétrico, e a unidade de medida é expressa em $m g L^{-1}$.

A divisão dos sólidos é feita por tamanho, por ser uma divisão prática. Todavia é possível afirmar que, partículas de menores dimensões, capazes de passar por um 
papel de filtro de tamanho especificado, correspondem aos sólidos dissolvidos, enquanto que as de maiores dimensões, retidas pelo filtro, são consideradas sólidos em suspensão (VON SPERLING, 1996).

Nas figuras abaixo é possível observar a média dos valores obtidos para os sólidos dissolvidos (Figura 2) e suspensos (Figura 3).

Figura 2: Valores para o parâmetro de sólidos dissolvidos.

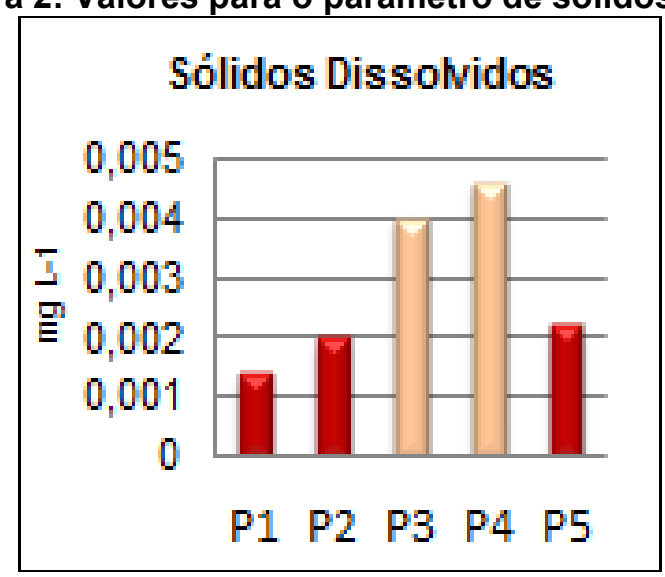

Figura 3: Valores para o parâmetro de sólidos suspensos.

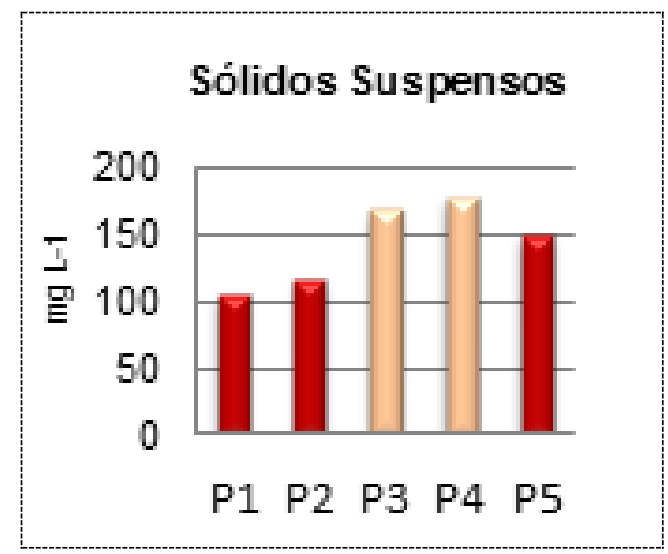

Já na Figura 4 é possível observar a diferença na cor da água, no parâmetro sólidos dissolvidos, para amostras derivadas do Basalto (a) e Depósitos Sedimentares (b). 
Figura 4: Amostra em Basalto (a) e Depósitos Sedimentares (b).

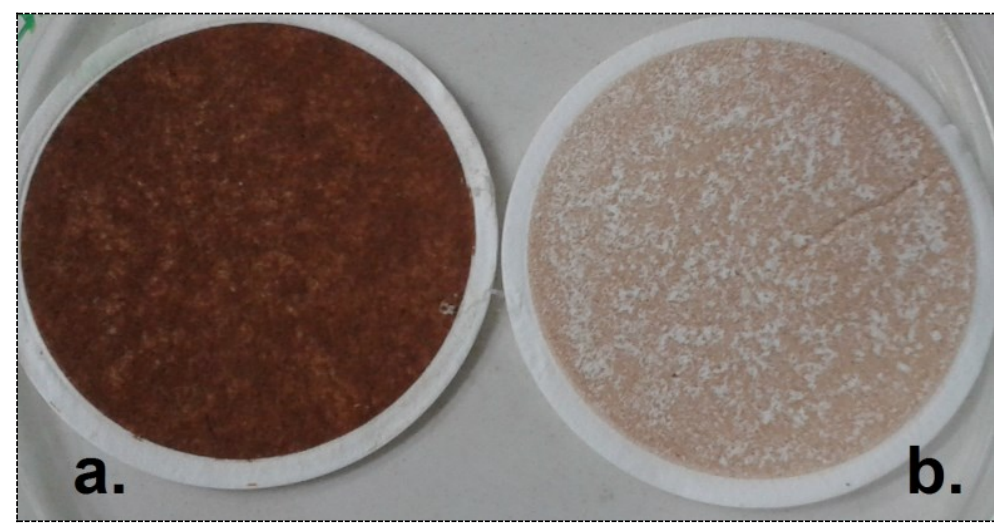

\section{CONCLUSÃO}

Conforme apresentado, para os parâmetros de sólidos dissolvidos e suspensos, os maiores valores foram obtidos nos pontos 3 e 4 , alocados sobre os Depósitos Sedimentares, ou seja, com solos do tipo Latossolo Vermelho e Argissolo, ambos de textura média. $E$, as menores concentrações nos pontos 1 e 2, pertencentes ao Basalto, sobre solos Latossolo Vermelho (textura argilosa) e Nitossolo. Em relação ao ponto 5, este apresenta resultados mais elevados que os dos pontos 1 e 2, mesmo ambos estando sobre o Basalto, todavia isso se justifica por sua localização, a jusante da bacia.

Desse modo, levando em consideração que todos os pontos são de mesmo uso da terra, e dando destaque aos fenômenos naturais, a geologia e ao material resultante desta - os solos - é possível concluir que há influência na qualidade da água, seja pelo aspecto visual ou pela questão da adequabilidade ao consumo humano.

Ou seja, existe grande influência dos solos, principalmente devido à questão de sua textura, uma vez que Latossolo Vermelho (textura média) e Argissolo são mais suscetíveis à erosão e a lixiviação do que solos do tipo Latossolo Vermelho (textura argilosa) e Nitossolo. Sendo assim, cabe ressaltar que deve se pensar em um manejo adequado às questões de uso e ocupação da terra, pela sociedade, uma vez que os 
fenômenos naturais, a estrutura dos solos, em si já colaboram com o aumento desse parâmetro, em eventos pluviométricos, e, atividades não planejadas acelerariam o aumento dessas partículas no corpo hídrico.

\section{REFERÊNCIAS}

APHA. American Public Health Association. Standard Methods for the Examination of Water and Wastewater. American Public Health Association. Washington, D.C., 1998, 1600 p.

BERTONI, J.; LOMBARDI NETO, F. Conservação do solo. 4. Ed. São Paulo: Ícone, 1999, p. 68-72.

BONIFÁCIO, C. M. Avaliação da fragilidade ambiental em bacias hidrográficas do alto vale do rio Pirapó, Norte do Paraná: Proposta metodológica. 2013. 110p. Dissertação (Mestrado em Geografia) - Departamento de Geografia. Universidade Estadual de Maringá.

BORSATO, F. H.; MARTONI, A. M. Estudo da Fisiografia das Bacias Hidrográficas Urbanas no Município de Maringá - Estado do Paraná. Acta Scientiarum Human and Social Sciences, UEM, Maringá, v.26, n. 2, p.273-285, 2004.

BRAGA, B.; HESPANHOL, I.; CONEJO, J. G. L.; BARROS, M. T. L.; SPENCER, M.; PORTO, M.; NUCCl, N.; JULIANO, N.; EIGER, S. Introdução à Engenharia Ambiental: o desafio do desenvolvimento sustentável. $2^{\mathrm{a}}$ Ed. São Paulo: Person Prentice Hall, 2005.

MELLO, W. S. Análise Ambiental da Bacia Hidrográfica do Ribeirão Maringá com Apoio de Imagens de Satélite. 2009. 37p. Monografia (Especialização em Gestão de Recursos Hídricos) Departamento de Engenharia Química. Universidade Estadual de Maringá, Maringá.

SALA, M. G. Análise da Fragilidade Ambiental na Bacia do Ribeirão Maringá - PR. 2005. 164p. Dissertação (Mestrado em Geografia) - Departamento de Geografia. Universidade Estadual de Maringá, Maringá.

SCHNEIDER, R. M. Qualidade da Água e Deposição de Sedimentos em Trechos da Bacia Hidrográfica do Rio Pirapó Representados pelo Ribeirão Maringá. 2009.

150p. Tese (Doutorado em Engenharia Química) - Departamento de Engenharia Química. Universidade Estadual de Maringá, Maringá.

TARELHO JUNIOR, O.; BONIFÁCIO, C. M.; TAVARES, C. R. G. Monitoramento da qualidade da água nas bacias do Paranapanema III e IV: parâmetros físicos. In: IX Fórum Ambiental da Alta Paulista, 2013, Tupã-SP. Fórum Ambiental da Alta Paulista. Tupã: ANAP, 2013. v. 9. p. 378-391.

VON SPERLING, M. Introdução à qualidade das águas e ao tratamento de esgoto: Princípios do tratamento biológico de águas residuárias. $2^{\mathrm{a}}$ ed. revisada. Vol. 1. Belo Horizonte. Universidade Federal de Minas Gerais, 1996. 